\title{
OBSERVATION OF AN OSCILLATING MAGNETIC FIELD SHELL AT THREE LOCATIONS
}

\author{
L. J. Cahil1, 1 M. Suglura, 2 N. G. Lin, 1 R. L. Arnoldy, 3
}

S. D. Shawhan, 4 M. J. Engeb retson, 5 and B. G. Ledley 2

Abstract. On July 14, 1982, 1830-1930 UT, a complex magnetic pulsation event was observed near the magnetic shell $\mathrm{L} \sim 4.5$ in the local afternoon. A large magnetic storm was in progress and had proceeded to the early recovery phase. The event began, at $1832 \mathrm{UT}$, with a sudden decrease in |B | and a brief, 120-s compressional pulsation. A transverse pulsation then developed with period near 180-s and amplitude about $5 \mathrm{nT}$. Near 1850 UT this pulsation decreased abruptly and was replaced by a 44-s transverse pulsation. By 1858 UT the 44-s pulsation was fading and a 240-s azimuthal pulsation developed rapidly. It slowly decreased in amplitude after 1910 UT and was gone by 1930 UT. The event was observed at the DE-1 satellite near the equator in both electric and magnetic field components and at the ground magnecic observatories Siple, Antarctica, and Roberval, Quebec, 15 to 30 west of $\mathrm{DE}-1$ and at opposite ends of an $L=4.2$ field line. The $180-s$ and $240-s$ pulsations were fundamental, toroidal, resonant oscillations of a magnetic field shell. Since the first 180-s pulsation started and stopped simultaneously at all three locations and the

corresponding magnetic pulsations remained in phase at all three locations, this is interpreted to be a large-scale toroidal oscillation of one resonant field shell. In the 240-s pulsation, DE- 1 was on one resonant shell and the ground stations, oscillating at 220 s period, were on another lower $L$ value shell. The $44 \mathrm{~s}$ pulsation, also transverse at $D E-1$, was weaker on the ground and poorly correlated with the DE-1 measurements, suggesting that it was more localized in azimuth.

\section{Introduction}

The DE-l satellite offers a new vantage point for observing hydromagnetic waves in the magnetosphere. At launch in August 1981, apogee of the elliptical orbit was at $4.65 \mathrm{R}_{\mathrm{E}}$ over the north pole; by July 1982, apogee had precessed to the equator. The orbit plane at this time, shown schematically in Figure 1, was essentially parallel to geographic meridian planes and, near the equator, the trajectory closely followed an inner magnetosphere magnetic field shell ( $L$ 4.5) for a few hours. Since some hydromagnetic waves propagate along the field lines this trajectory allowed observation of such waves for an extended

\footnotetext{
$1_{\text {School }}$ of Physics and Astronomy, University of Minnesota.

2NASA Goddard Space Flight Center.

3 Department of Physics, University of New Hampshire.

${ }^{4}$ Department of Physics and Astronomy, University of lowa.

5 Department of Physics, Augsburg College.
}

Copyright 1984 by the American Geophysical Union.

Paper number $4 \mathrm{A0010}$.

0148-0227/84/004A-0010\$05.00 period. Our earlier studies of hydromagnetic waves in space have been conducted on satellites with elliptical orbit planes near to the equatorial plane so that the trajectory passed rapidly across field lines near $L=5$ [Patel and Cahill, 1964; Lin and Cahill, 1976; Engebretson and Cahill, 1981]. The geostationary satellites, such as the ATS series, provided a much better location for wave observation at an almost fixed location on a field line 24 hourg a day and they have been extensively used in a wide variety of hydromagnetic wave studies (for example, Hughes et a1. [1978]). In practice, the DE-1 satellite slices across closely spaced L shells as it moves from south to north, traveling for example from $L=5$ to $L=4$ ip about 2 hours while it moves from -10 to +10 in magnetic latitude. In the summer of 1982 the DE-1 apogee was in the afternoon quadrant of the magnetosphere, near the plasmapause, providing an excellent opportunity to study hydromagnetic waves with periods from $1 \mathrm{~s}$ to a few hundred seconds during both magnetically quiet and disturbed intervals.

One of the objectives of the hydromagnetic wave study was a comparison of the wave characteristics (frequency, amplitude, polarization) with those predicted by theory. Sugiura and Wilson [1964], suggested that long period $(\tau>100$ s) magnetic pulsations, long observed at ground observatories, were standing waves on the earth's magnetic field lines, analogous to the waves on strings. Later Hasegawa [1969] offered a source mechanism for certain long period resonant waves, the drift mirror instability ariaing from proton pitch angle anisotropy in the high $\beta$ plasma during magnetic storms. More recently Chen and Hasegawa [1974] and also Southwood [1974] have presented a comprehensive theory where resonant waves are caused by surface waves on the magnetopause and have included predictions of wave polarization and of the variation of wave amplitude with $L$ value.

We have taken as an example of our current pulsation study a complex wave event observed on July 14, 1982, during the early recovery phase of a magnetic storm. In addition to the DE-1 magnetometer observations, measurements from an electric field double probe on the same satellite are available and, for this event, ground magnetometer observations near the foot of the magnetic field line through the satellite were obtained. Thermal plasma measurements from a retarding ion mass spectrometer were also obtained on $\mathrm{DE}-1$, and these measurements will be discussed in a separate paper [Johnson et al., unpublished manuscript, 1984].

\section{Observations}

The spacecraft was spin stabilized with spin axis perpendicular to the orbit plane, and the spin period was $6 \mathrm{~s}$. The magnetometer was a three axis flux gate instrument with one axis parallel to the spin. Sixteen vector samples were recorded each second. There were three dynamic ranges of operation of the magnetometer. Data described 


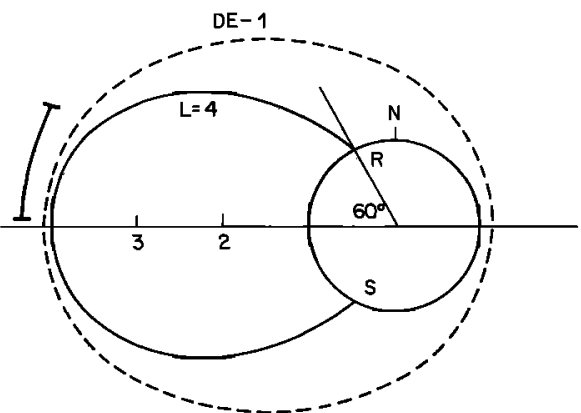

Fig. 1. Schematic view of the DE-1 orbit (dashed line) in June 1982 in relation to an $\mathrm{L}=4$ field line joining Siple (s) and Roberval (R) magnetic pulsation observatories. The location of the present observations is shown by a bar.

here were obtained in the most sensitive range with $\pm 0.02 \mathrm{nT}$ resolution. A detailed description is contained in Farthing et al. [1981]. The electric field instrument had a $200 \mathrm{~m}$ double probe $\left(E_{x}\right)$ perpendicular to the spin axis and a $9 \mathrm{~m}$ double probe $\left(E_{z}\right)$ along the spin axis. Operation of the instrument is described in the work of Shawhan et al. [1981]. Ground observations of magnetic pulsationg were obtained at Siple, Antarctica, $76^{\circ} \mathrm{S}$ latitude, $84^{\circ} \mathrm{W}$ longitude, and Roberval, Canada, $49^{\circ} \mathrm{N}$ latitude, $72^{\circ} \mathrm{W}$ longitude. Large search coif magnetometers were used sensitive to $\mathrm{dB} / \mathrm{dt}$ in the range 0.001 to $10 \mathrm{~Hz}$. The instrument is described in the work of Taylor et al. [1975]. The observations to be described were obtained during the early recovery phase of a large magnetic storm. Dst was $-338 \mathrm{nT}$ in the main phase 17 hours earlier and below $-120 \mathrm{nT}$ during the event.

Figure 2 shows that the magnetic field is inflated at the time of the measurements. The inclination angle is 6 greater thgn was predicted at 1830 UT near the equator and 12 greater at 1920 UT, 15.5 magnetic latitude, indicating field lines stretched outward away from the earth. The declination angle shows the field line to be also stretched towards the tail, 1 west of predicted at $1830 \mathrm{UT}$ and 3 at $1920 \mathrm{UT}$. The field magnitude is equal to the predicted value at $1830 \mathrm{UT}$ while a negative $\Delta B$ is expected for the inflated inner magnetosphere field during the early recovery phase of a large magnetic atorm. This configuration indicates that the major portion of the storm time ring current is below the spacecraft at $L=4.5$ and that the spacecraft is near the location where equatorial $\triangle B$ changes from negative at lower $\mathrm{L}$ values to positive at higher $\mathrm{L}$ values. This polarity results because the magnetic field from the westward current below the satellite is positive or northward. The average $\Delta B$ distribution in the magnetosphere both near the equator and at higher latitudes has been given by Sugiura and Poros [1973].

Figure 3 shows the magnetic fluctuations in geomagnetic opherical components $\Delta B_{r}, \Delta B_{\theta}$, and $\Delta B$ where a model vector field [Langel et al., 1980] has been subtracted from the observed field components. $B_{\theta}$ is antiparallel to the dipole magnetic field at the equator, Br points radially outward, and $B$ points east. There are three distinct and separate wave events in the one hour interval shown in Figures 2 and 3 . The first event, from 1832 to 1850 UT starts out with strong pulsations in $B$ and I (in Figure 2) between 1832 and 1838 , characteristic of a compressional wave. The period is about $120 \mathrm{~s}$ although an apparent phase shift occurs at 1836 UT. The wave is also seen clearly in $\Delta B$ and $\Delta B_{r}$ (Figure 3 ). As the compressional wave dies out, a wave in $B_{\text {( }}$ (D) grows, from 1834 to $1848 \mathrm{UT}$, with $\sim 180$ - $\$$ period. There are weak $B_{r}$ and $B_{\theta}$ components to this wave and a shorter period wave, 30-50 s, is superimposed on the 180-s wave. Note that the compressional wave has $\Delta B$ and $\Delta I$ approximately in phase for the first two cycles, 1832-1836 UT, that is $\Delta B$ decreases with $\Delta I$ for the first two pulses. A decrease in $\Delta I$ in the northern hemisphere usually corresponds to a compression, while a $\Delta B$ decrease near the equator corresponds to an inflation of the field. An inward propagating compressional wave originating at the magnetopause should have an increase in $\Delta B$ together with a decrease in $\Delta I$ [Lin and Cahill, 1976].

The second event is a transverse oscillation in

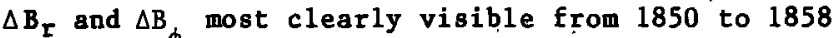
UT but present from 1836 to 1850 with perhaps somewhat different period and until 1900 UT with reduced amplitude. The period is $44 \mathrm{~s}$ in the clearest segment and the amplitude in $B$ grows from $3 \mathrm{nT}$ to $5 \mathrm{nT}$ from 1850 to $1855 \mathrm{UT}$ while the $\mathrm{B}_{\mathrm{r}}$ amplitude decreases.

The third event is again of longer period, $240 \mathrm{~s}$, and is primarily in $\Delta B_{\text {, }}$ (D). It grows in amplitude from 1858 UT $(5 \mathrm{nT})^{\phi}$ to $1906(10 \mathrm{nT})$ and then decreases to $3 \mathrm{nT}$ by $1920 \mathrm{UT}$. The $\Delta \mathrm{B}_{\mathrm{r}}$ component is half the amplitude of $\Delta B_{\phi}$, or less,

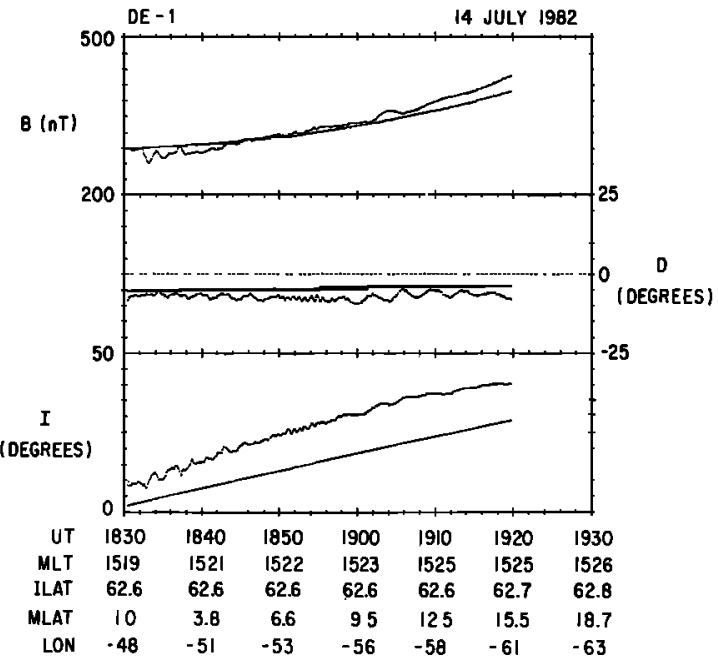

Fig. 2. Record from the DE-1 magnetometer July $14,1982,1830-1920$ UT. $B$ is the field magnitude in nT. The observed magnitude is the fluctuating line and the predicted MAGSAT magnitude is the smooth line [Langel et al., 1980]. Declination (D) is the angle of the fieid to the magnetic meridian plane (positive eastward) and inclination (I) is the dip angle in degrees of the field below the local horizontal plane (positive in northern hemisphere). Universal time, local time, altitude," geographic latitude, geographic longitude, magnetic local time, invariant latitude, and magnetic latitude are given on the bottom. 
and leads $\Delta B_{\text {}}$ in phase by about $90^{\circ}$, as can be seen in Figute 3 between 1900 and 1904 UT. Note the pulsations in $\Delta B$ are strongest between 1900 and 1906 UT and in phase with $\Delta B_{\phi}$. Also there are low amplitude, short period pulsations in $B_{\phi}$, about $20 \mathrm{~s}$, from 1910 to 1920 UT.

The record from the electric field probe in Figure 4 shows clearly an oscillation with period $180 \mathrm{~s}$ in $\mathrm{E} \perp$ that grows to a maximum amplitude of 5 $\mathrm{mV} / \mathrm{m}$ at 1842 UT then decreases to $1 \mathrm{mV} / \mathrm{m}$ by 1852 UT. Then another oscillation, this time of a 240-s period, grows to $5 \mathrm{mV} / \mathrm{m}$ at 1905 UT and decreases to $1 \mathrm{mV} / \mathrm{m}$ by $1930 \mathrm{UT}$. In the transition period between these two waves near 1854 a weak higher frequency wave is observed, perhaps corresponding to the 44-s pulsation in $\Delta B, E \perp$ is essentially in the radial direction since ${ }^{\phi}$ it $i s$ derived from probes rotating in the orbit plane. This orbit plane component has been separated as $E \perp$, perpendicular to $B$, and $E \|$, parallel to $B$. $E_{z}$ is from the shorter boom along the spin axis and perpendicular to the orbit plane. $E \perp$ is approximately in the $\Delta B_{r}$ direction and $E_{z}$ in the $\Delta \mathrm{B}_{\phi}$ direction.

${ }_{0}$ In a hydromagnetic wave the E perturbation is $90^{\circ}$ to the $B$ perturbation so $\mathrm{E} \perp$ should correspond to $\Delta B$ and $E_{z}$ to $\Delta B_{r}$. The growth in the amplitude of $\mathrm{E}^{\phi}$ from 1855 to 1905 is similar to the growth of $\Delta B_{\phi}$ supporting the identification of $E \mid$ with $\mathrm{B}_{\phi}$. Between 1835 and 1850 the $\mathrm{E} \perp$ and $\Delta \mathrm{B}_{\phi}$ amplitude profiles are quite different, however. The $B_{\phi}$ amplitude grows steadily from 1835 to 1845 UT while $E \perp$ reaches a maximum at 1942 . This suggests that $B_{\phi}$ increases while moving north from a node at the equator. Also the relatively small amplitude of the 44 second $E \perp$ component, 1850-1900 UT, does not agree with that of $\Delta \mathrm{B}_{\phi}$. The $E_{z}$ amplitude behaves similarly to $E$, 1830$1930 \mathrm{UT}$, but it is roughly three times larger in amplitude and thus the parallel between $E_{z}$ and $\Delta B_{r}$ is lesg convincing. Since the $E_{z}$ record is from the short boom its amplitude may be less reliable because of Debye sheath effects on short

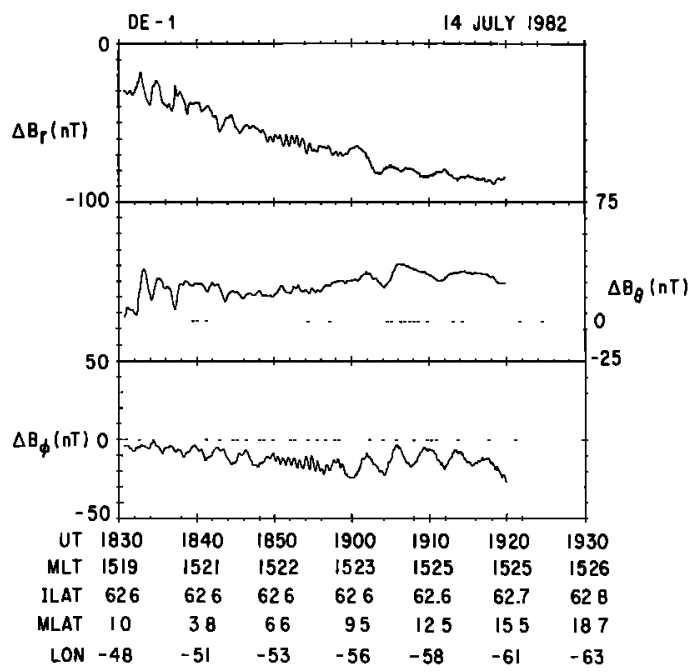

Fig. 3. Same as Figure 2 but in geomagnetic spherical componente. $B_{r}$ is positive radially outward, $B_{\theta}$ is positive southward, and $B_{\phi}$ is positive eastward. The MAGSAT model field has been subtracted from the observed components leaving $\Delta \mathrm{B}_{r}, \Delta \mathrm{B}_{\theta}$, and $\Delta \mathrm{B}_{\phi}$.

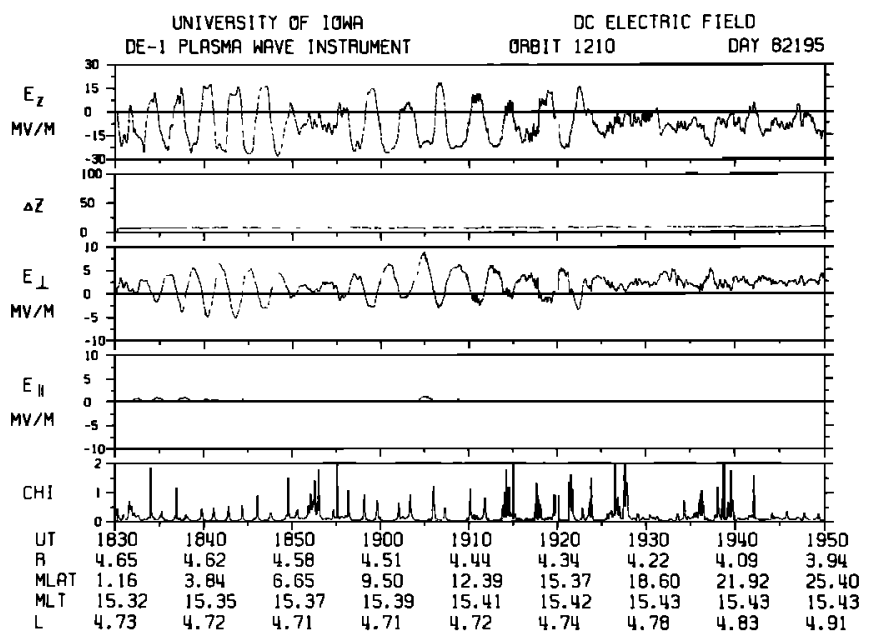

Fig. 4. Electric field record from $D E-1 . E_{2}$ in $\mathrm{mV} / \mathrm{m}$ is antiparallel to the satellite spin axis, nearly east-west. This measurement is from the short boom and is less reliable than the measurements from the long boom. The long boom is perpendicular to the spin axis and measures the component of $E$ in the spin plane, approximately the magnetic meridian plane. The spin plane $E$ vector is used to obtain the components E L, perpendicular to the magnetic field and approximately in the radial direction, and $E \|$, parallel to the magnetic field. CHI is a measure of the uncertainty in the $E \perp$ component and $\Delta Z$ in the $E_{z}$ component. Positive $E \perp$ corresponds to positive $E_{r}$ (outward) and positive $E_{z}$ to $E_{\phi}$ (eastward).

booms in low density plasmas [Cauffman and Maynard, 1974]. Note also that the irregular shorter period waves ( $20 \mathrm{~s}$ ) superimposed on the $\Delta \mathrm{B}_{\phi} 240$ second pulsation from 1910 to 1920 are seen on $E \perp$ in the same interval. There a few small pulsations in E\|, lmV/m; they occur in close correlation with the more prominent pulsations in B (Figure 2) suggesting a connection with the compressional pulsations.

Figure $5 a$ shows a record of $B_{x}$ (north), $B_{y}$ (west), and $B_{z}$ (up) fluctuations recorded on the Roberval search coil magnetometer. There are clearly two wave events in $B_{x}$ and $B_{y}$. One, from 1832 to $1852 \mathrm{UT}$, atarts with a wave of $\sim 150 \mathrm{~s}$. In the second, 1852 to 1910 UT, the period is about $220 \mathrm{~s}$. The $B_{x}$ wave from 1852 to 1905 UT grows in amplitude, as do $\Delta B_{\phi}$ and $E \perp$ at $D E-1$, so we identify $B_{X}$ with $\Delta B_{\phi}$. At Roberval $B_{z}$ is aligned opposite to the local $B$ vector; the pulsations are relatively weak in the $B_{z}$ record but have similar structure to those in $B_{x}$ and $B_{y}$. There is some indication of a weak 40-s fluctuation near $1850 \mathrm{UT}$ in $B_{x}$ and in $B_{y}$. The long period $B_{y}$ pulsations are about one half the amplitude of those in $B_{x}$, and between 1855 and 1905 the wegtward $B_{y}$ peaks lead northward $B_{x}$ peaks by about 90 in phase, indicating right hand polarization.

Figure $5 b$ is the siple record of this event with $B_{x}$ (north), $B_{y}$ (west), and $B_{z}$ (down). The signal amplitude at siple is about one third that at Roberval. The $B_{y}$ signal amplitude is relatively larger than $B_{x}$ at Siple, while at Roberval the reverse is true. From 1855 to 1905 UT $B_{Y}$ (west) leads $B_{x}$ (north) by $90^{\circ}$ indicating $1 \mathrm{eft}$ 

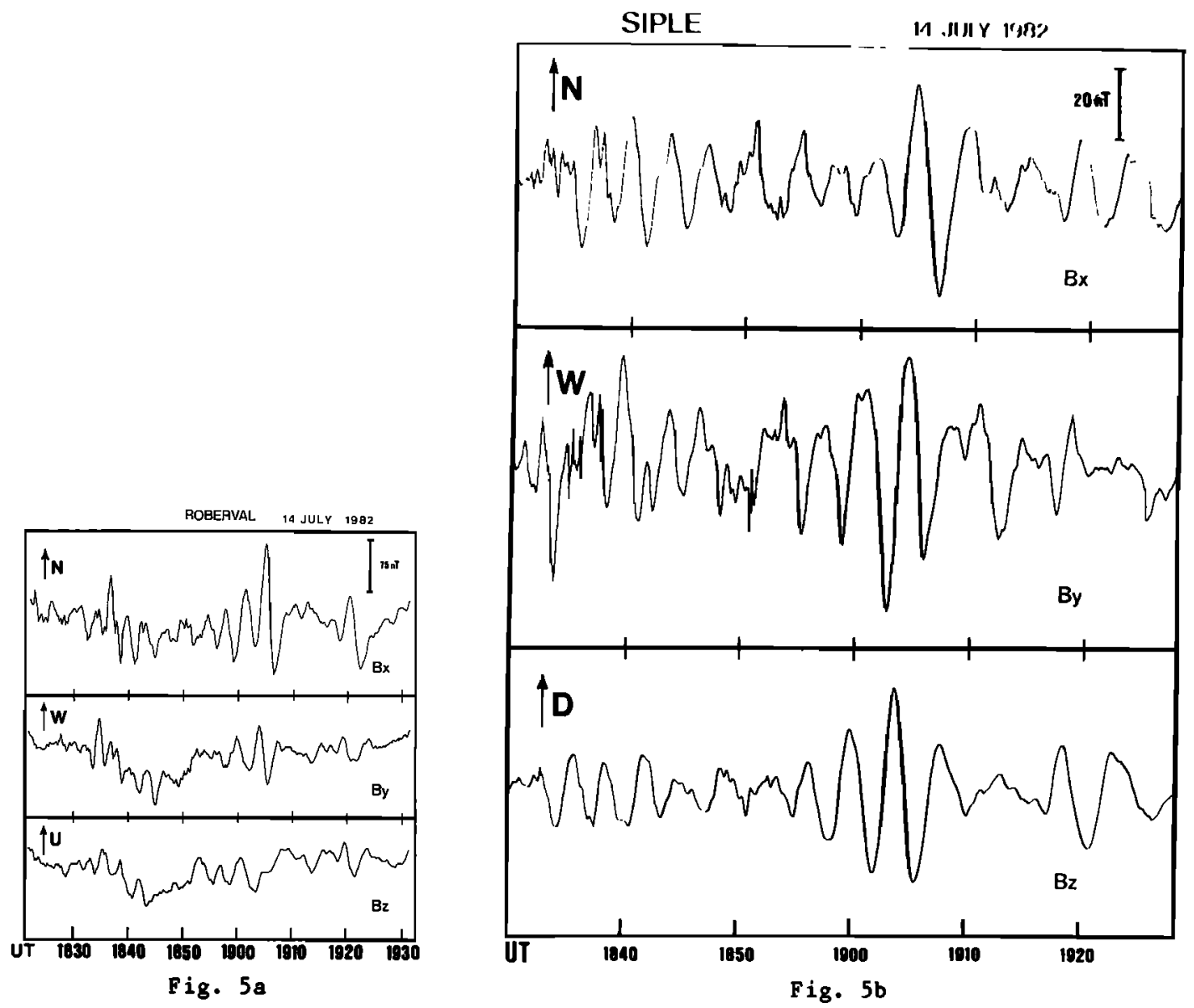

Fig. 5. (a)Record from the Roberval search coil magnetometer. $B_{x}$ is positive north, $B_{y}$ is positive west, and $B_{z}$ is positive up. The original data from the search coil magnetometer were $\mathrm{dB} / \mathrm{dt}$ in $\mathrm{nT} / \mathrm{s}$. The signals shown have been integrated and averaged over $6 \mathrm{~s}$. The approximate scale of the $\mathrm{B}$ components is shown by a $75 \mathrm{nT}$ bar. (b)The Siple record is similar to Figure $5 a$, but note the smaller signal amplitude. $B_{z}$ is positive down.

hand polarization. The $B_{z}$ gignal is comparable to $B_{x}$ in amplitude and similar in structure. The $B_{x}$ and $B_{2}$ signalo near 1840 UT are quite similar to the EI signals at $\mathrm{DE}-1$. The shorter period signal, 1848-1855 UT, can be seen in all three components.

Power spectral density for the DE-1 waves for the interval 1844-1909 UT, including portions of the three wave events, is shown in Figure 6 . The dominant peak near $0.005 \mathrm{~Hz}$ is primarily due to the $B_{\phi}$ fluctuations from 1855 to 1909 UT. The shorter period fluctuations show up as a weaker peak at $0.023 \mathrm{~Hz}(43.5 \mathrm{~s})$. The $B_{T}$ and $B_{\theta}$ peaks near $0.005 \mathrm{~Hz}$ are smaller than the $B_{\phi}$ peak. The $0.023-\mathrm{Hz}$ peak can be seen in $B_{r}$. The spectral history of the $D E-1 \quad B_{\phi}$ component is shown in Figure 7. Time is plotted along the horizonta1 axis, frequency from 0 to $0.063 \mathrm{~Hz}$ vertically, and spectral power density is shown by a logarithmic gray scale. The dominant activity starts in a band between 0.005 and $0.01 \mathrm{~Hz}$ at 1830 and increases in intensity while decreasing slightly in frequency to 1912 UT. There is a gap in power near $0.005 \mathrm{~Hz}$ between 1850 and 1855 ; this is where the $0.023 \mathrm{~Hz}$ wave can be seen as a narrow dark band.

\section{Discussion}

The dominance of the $B_{\phi}$ fluctuations in Figure 3 and the good correlation with similar fluctuations in $B_{x}$ at Siple and Roberval indicate that the events are toroidal mode standing wave oscillations of magnetic field shello near $L=$ 4.5. The long period of the near $180-8$ pulsations and the steadily rising amplitude of $B_{\phi}$ from 1835 to 1845 UT suggest that these are fundamental, half wavelength resonances with a node in $B_{\phi}$ and antinode in $E_{r}$ at the equator. We now proceed to examine this identification in more detail.

The phase difference between $E_{r}(E \perp)$ and $B_{\phi}$ confirms that the wave is the fundamental mode. Comparison of the phase of $E_{r}$ and $B_{\phi}$ in Figures 3 and 4, for example near 1840 or $1900 \mathrm{UT}$, shows that $E_{r}$ leads $B_{\phi}$ by about $90^{\circ}$. A schematic view of the fundamental and second harmonic field line resonance in Figure 8 shows that this is the correct phase relation for the fundamental but not for the second harmonic [Singer et al., 1982]. In each case in the figure the field line is shown at zero displacement (maximum plasma velocity) and at maximum displacement (zero plasma velocity) one quarter period later. Above the equator the 
plasma velocity at maximum is westward in each case corresponding to $E_{r}$ positive (outward) since $\bar{E}_{T}=-\bar{v} \times \bar{B}_{0}$. For the fundamental a maximum in $B_{\phi}$ eastward occurs $90^{\circ}$ after a maximum in $E_{r} \cdot E_{r}$ leads $B_{\phi}$ by $90^{\circ}$. For the second harmonic, the phase of $B_{\phi}$ is changed by 180 and $B_{\phi}$ leads $E_{r}$. We can use the measured $E_{r}$ and $B_{o}$ to calculate the plasma velocity for this wave since $V=E_{r} / B_{O}$. Taking $E_{x}$ as $5 \mathrm{mV} / \mathrm{m}$ and $B_{o}$ as $300 \mathrm{nT}, V=5 \mathrm{x}$ $10^{-3} / 3 \times 10^{-7} \sim 17 \mathrm{~km} / \mathrm{s}$. This may be compared with the plasma velocity determined from the thermal plasma measurements [Johnson et al., unpublished manuscript, 1984]. Also the maximum field line displacement is $\mathrm{E}_{r} / \mathrm{B}_{\mathrm{o}} \omega$ or about $540 \mathrm{~km}$. The observed pulsations are not pure toroidal oscillations; there are pulsations in the radial component as well as the azimuthal component. For example, a close examination of Figure 3 between 1900 and 1905 UT shows that $\Delta B_{r}$ pulsations lead $\Delta \mathrm{B}_{\phi}$ by about $90^{\circ}$. In order to compare the phase between the two transverse components at each of the observation points the data were first filtered to focus on the long period waves. Figure 9 shows the $\mathrm{DE}-1$ data in a passband of 100-500 8; similar filters were also applied to the Siple and Roberval data. Polarization hodograms for the interval 1843-1848 UT are shown in Figure 10a. We see that the sense of polarization looking in the direction of the magnetic field is generally left handed at each location although there are some irregularities in the traces and the Siple polarization is almost linear. Other intervals from 1833 to 1853 UT also show principally left hand polarization.

Polarization hodograms in another interval, 1903-1909 UT, Figure 10b show right-hand polarization at Roberval and $D E-1$ but left hand at Siple. This suggests that Roberval and $D E-1$ are on the same field shell but that Siple is on a higher shell (further out in the magnetosphere). In the theory of Southwood [1974] and Chen and Hasegawa [1974] Kelvin-Helmholtz surface waves on the magnetopause are the source of the transverse standing wave oscillations. There is maximum

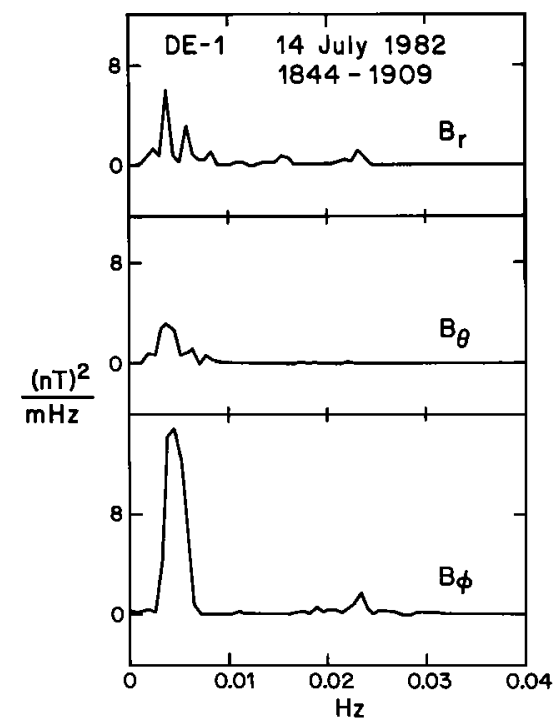

Fig. 6. Power spectral density for the central portion of the wave events.

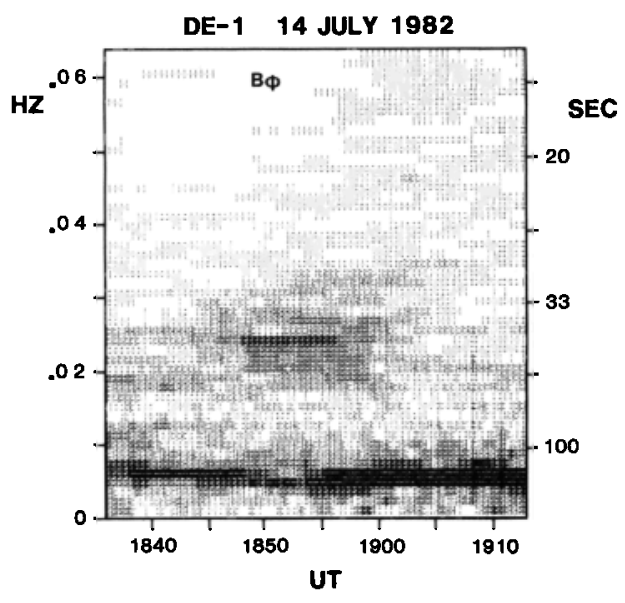

Fig. 7. Dynamic power spectrum for the $D E-1 B_{\phi}$ wave between 1835 and 1912 UT.

amplitude at some $L$ value across which the oscillation changes in polarization sense. In the afternoon hours the change is from left hand at higher I (latitude) to right hand at lower L [Hughes and Southwood, 1976b]. Note in Figure 10b that the mgjor axis of the polarization ellipse rotates 90 between the equator and Roberval (somewhat less than 90 at Siple). We had earlier in this report identified $B_{x}$ (north) in the ground records with $B_{\phi}$ (east) at $\mathrm{DE}-1$. The east-west motion of the resonant field lines in the ionosphere carries along the plasma there. At about $100 \mathrm{~km}$, at the peak in ionospheric conductivity, the positive ions are retarded by collisions while the electrons continue to move with the field

line. This constitutes an ionospheric current (I) which in turn causes a north-south magnetic field oscillation on the ground below. Figure 11 shows the relations between $E, V$ and $I$ in the ionosphere and $\mathrm{B}_{X}$ below. So $\mathrm{B}_{\phi}$ at $\mathrm{DE}-1$ rotates to $\mathrm{B}_{X}$ on the ground and $B_{r}$ to $B_{y}$. The northeast orientation of the polarization ellipse, at stations near $\mathrm{L}=4$ in the northern hemisphere in the afternoon as well as left-hand polarization on the highlatitude side of the resonance maximum has been reported in ground level studies by Fukunishi and Lanzerotti [1974].

We studied the polarization sense of the 40-8 pulsations in the same way. Figure 12 shows a filtered version (20-to 60-s period) of the $D E-1$
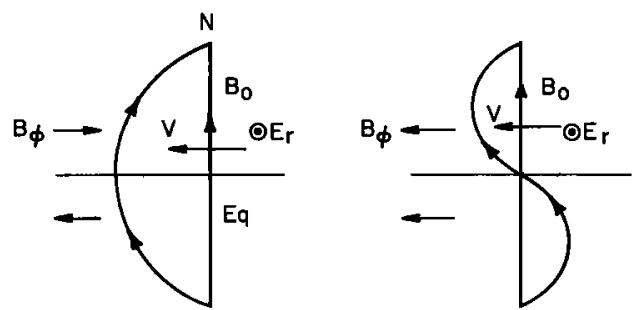

Fig. 8. Schematic view of the toroidal half wave fundamental and second harmonic resonance oscillation. $B_{0}$ is the field line at rest. $E_{r}$ points radially outward. Positive $B_{\phi}$ represents the eastward component of displaced field line direction. 


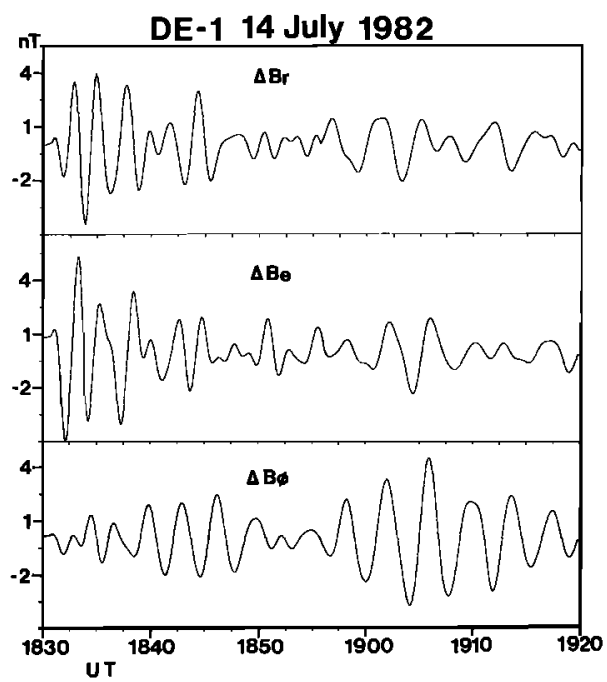

Fig. 9. The DE-1 magnetometer record, 1830-1920 UT, filtered at $100-500$ s band pass.

data. Two hodograms of the DE-1 data in Figure 13 show that the polarization is initially right hand but that it becomes more nearly linear in the second interval. Filtered versions of the Siple and Roberval data (not shown here) confirmed weak amplitude in this band and correlation with the $\mathrm{DE}-1$ waves was low. Hodograms (not shown) were very irregular with no consistent sense of polarization. We conclude from consideration of the polarization ellipses of event 1 that Siple, DE-1 and Roberval are all on the same oscillating field shell, and because they are all left-hand polarized they are on the high $L$ value side of the resonance maximum. The nearly linear siple hodogram indicates siple is close to the resonance maximum. For event 3 , Roberval and DE-1 appear to be on the low $\mathrm{L}$ value side of a resonance while Siple is on the high side. For event 2, the $44 \mathrm{~s}$ pulsation, DE-1 is on the low side of resonance and, as the ellipse becomes linear, DE-l is close to the resonance maximum. The poor correlation of DE-1 waves with the 40 s ground waves suggesta that the ground stations are not close to being conjugate with the oscillating shell that $\mathrm{DE}-1$ is on or that the oscillation is of limited azimuthal extent.

A recent analysis of the pure toroidal mode oscillation in the magnetosphere, including realistic magnetosphere plasma densities and ionospheric conductances, provides a very useful model for comparison with our observations [Allan and Knox, 1979a, b; Allan, 1982]. In addition, the work of Hughes and Southwood [1976a, b] on the effects of the ionosphere on magnetic pulsations allows a model conversion of magnetosphere to ground pulsations.

In the classical standing wave picture of a toroidal half wave resonance the phase of $B_{\phi}$ and $\mathrm{E}_{\mathrm{r}}$ should be constant and with 90 phase difference from the equator down to the ionosphere. At first glance we might expect $B_{x_{0}}$ on the ground to be in phase with $E_{r}$ and thus $90^{\circ}$ out of phase with $B_{\phi}$. There should, however, be a node in $E_{r}$ in the infinite conductance of the ionosphere, while $B_{\phi}$ should have an antinode there. We examine the phase between $E_{r}, B_{\phi}$, and $B_{x}$ in Figure 14. First we note that $B_{x}$ (north) at Siple and Roberval are in phase, allowing for irregularities in the wave forms, from 1835 to 1910 UT. This provides a very strong argument that both stations are on the same toroidally oscillating half wave fundamental field shell during this interval. The oscillation period is obviously the same, so the stations are on the same resonant shell. Both stations remain in phase, so the oscillation must be the fundamental. Take for example an eastward field line
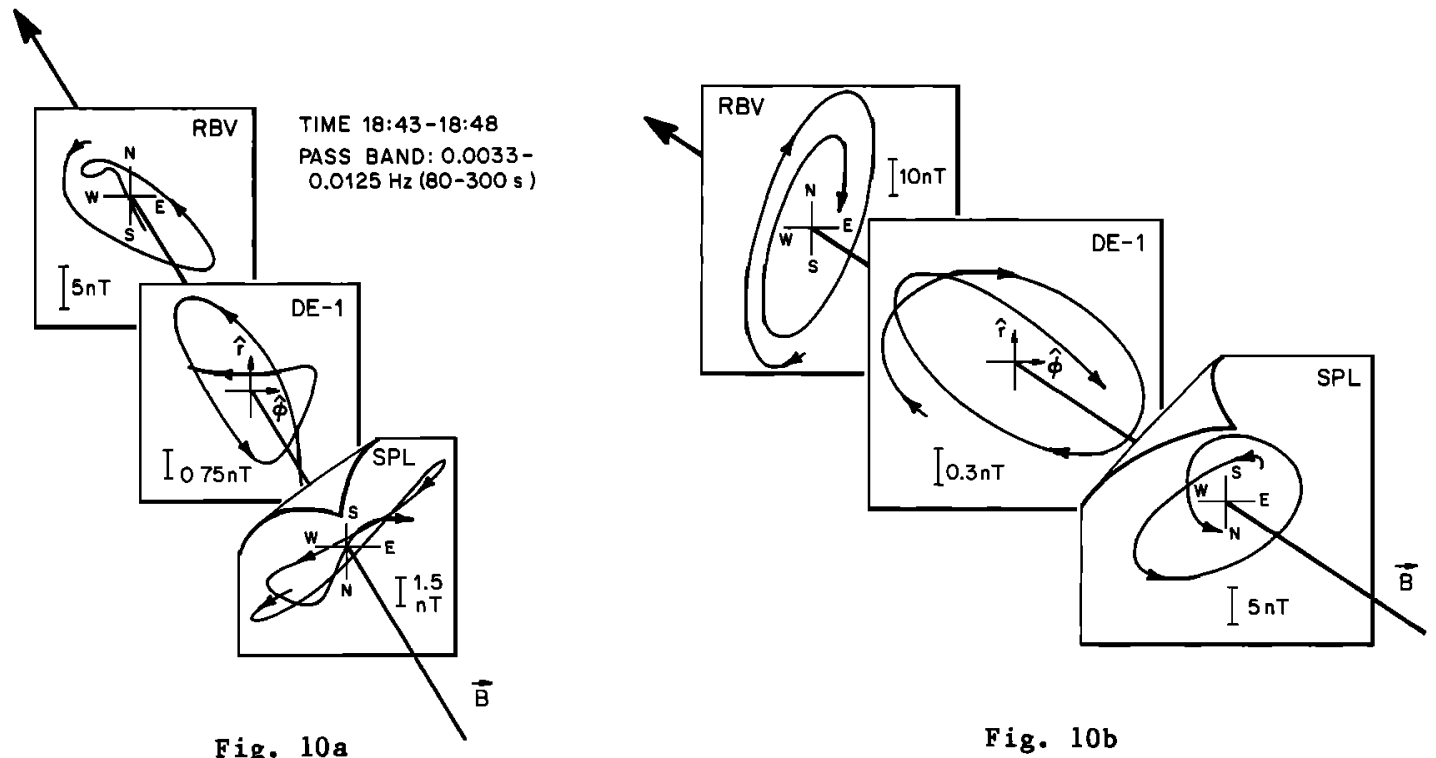

Fig. 10. (a)Polarization hodograms for the interval 1843-1848 UT plotted with filtered components data. Each view is of a plane perpendicular to the main field vector $\bar{B}$. $N, P$, and $S$ are each the outward direction in the magnetic meridian plane. In this case each observation is left-hand polarized. (b)Same as Figure 10a but for the interval 1903-1909 UT. The pass band is the same as for Figure 10a. 
motion of the fundamental mode producing eastward plasma motion in the ionosphere over both stations. This also produces a northward, in phase, $B_{x}$ at each station. The second harmonic field line motion would have eastward motion at one station and westward at the other leading to a $180^{\circ}$ phase difference in $B_{x}$.

The phase difference between $\mathrm{E}_{r}$ and $\mathrm{B}_{\phi}$ is $90^{\circ}$ as in the classical standing wave model but $B_{x}$ and $B_{\phi}$ are in phase from 1835 to 1850 UT. Disregarding at first the azimuthal separation, about 30 longitude, between $\mathrm{DE}-1$ and the ground stations, we consider the finite ionospheric conductivity resonance models of Allan and Knox [1979b]. In one case they show (Figure 5, their paper) for $\mathrm{L}=4$ and for a northern ionosphere height integrated conductivity of $10 \mathrm{~s}$ and southern ionosphere $3 \mathrm{~s}$, the amplitudes and phases of $E_{r}$ and $B_{\phi}$ along the field line from one ionosphere to the other. Unlike the constant phase in $B_{r}$ for the infinite ionospheric conductivity model, the phase of $\mathrm{E}_{\mathbf{r}}$ now is retarded, behind $E_{r}$ at the equator, as the wave approaches the ionosphere. At the bottom of both north and south ionospheres $E_{r}$ is in phase with $B_{\phi}$ at $D E-1$ as is $B_{x}$. Thus, considering the finite conductivity, the in phase relation between $B_{\phi}$ and $B_{x}$ in Figure 14 appears to be correct. The situation is different for event 3. At 1905 UT $B_{x}$ leads $B_{\phi}$ by about 90 but we note a changing phase difference between 1855 and 1905 UT. In this interval the period of the oscillation at siple and Roberval is $220 \mathrm{~s}$, while that at $\mathrm{DE}-1$ is $240 \mathrm{~s}$. DE-1 is on an oscillating shell of slightly longer oscillation period than that at Siple and Roberval.

We can also translate the $B_{\phi}$ and $E_{T}$ amplitudes seen at $\mathrm{DE}-1$ to the ionosphere using the model of Allan and Knox [1979b] and from the ionosphere to $B_{x}$ on the ground using the calculations of Hughes and Southwood [1976b]. We take $B_{\phi}$ as $5 \mathrm{nT}$ and $E_{r}$ a $5 \mathrm{mV} / \mathrm{m}$ at $\mathrm{DE}-1$. We note that the model has oimilar fields (but $1 \mathrm{nT}$ and $1 \mathrm{mV} / \mathrm{m}$ ) somewhat north of the equator. The model shows $E_{r}$ with no change from the equatorial value to the northern high conductivity ionosphere and with a factor 3 increase at the southern law conductivity ionosphere, giving $5 \mathrm{mV} / \mathrm{m}$ north and $15 \mathrm{mV} / \mathrm{m}$ south. $B_{\phi}$, however, increases by a factor 10 at both north and south ionosphere or to $50 \mathrm{nT}$ in our case.

In the calculations of Hughes and Southwood [1976b] the pulsation amplitude $B_{x}$ on the ground is reduced from that at the top of the ionosphere.

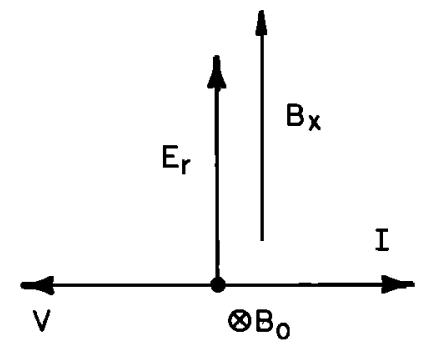

Fig. 11. Schematic view of the relation between plasma velocity $(v)$, electric field $\left(E_{r}\right)$, main magnetic field $\left(B_{0}\right)$, ionospheric current (I), and ground field perturbation $\left(B_{x}\right)$. We are looking down at the northern ionosphere and ground. Bo points down, I east and $B_{x}$ north.

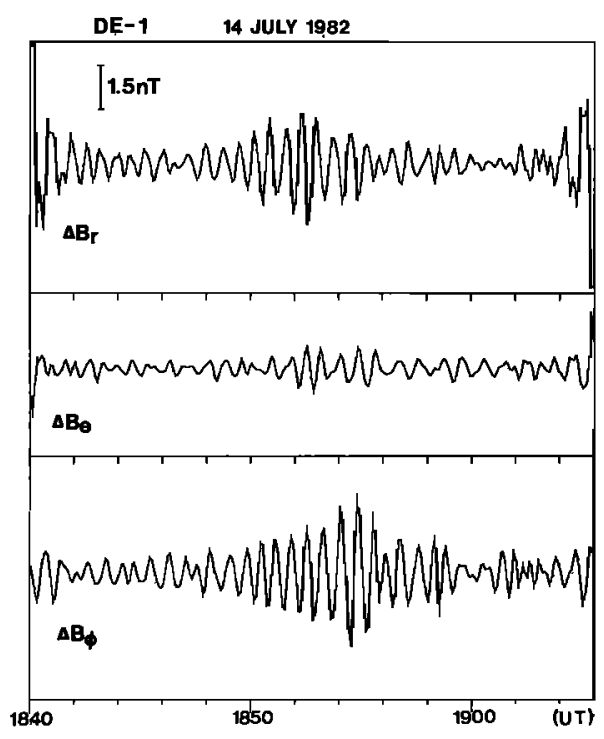

Fig. 12. Filtered DE-1 magnetometer data (1840-1905 UT) in a band pass for 20 to $60 \mathrm{~s}$.

The factor depends on the ratio of integrated Hall conductivity to Pedersen conductivity and on the scale of the resonant structure. We take their second case with latitudinal resonance scale about 1 for comparison. This case has $B_{x} \sim B_{\phi} / 2$ giving $25 \mathrm{nT}$ amplitude on the ground comparable to that observed at Roberval. At Siple, however, there appears to be twice as much attenuation between the top of the ionosphere and ground. Of course the ground signal amplitude also depends on how close the station is to the resonance peak. Siple may be further from the peak than Roberval.

Because of the finite conductivity the wave dissipates energy in the ionosphere. Greenwald and Walker [1980] have calculated the rate of energy dissipation in the ionosphere by Joule heating through the ionospheric Pedersen current, parallel to $E_{r}$, that flows in response to the wave electric field. They use $W=\left(E_{\text {rms }}\right)^{2} \Sigma_{p}$ where $E_{\text {rms }}$ is the wave amplitude and $\Sigma_{p}$ is the height integrated ionospheric Pedersen conductivity. For a large pulsation event $44 \mathrm{mV} / \mathrm{m}$ and $\Sigma_{p}=2.5 \mathrm{~s}$ they find $W=4.8 \mathrm{~mW} / \mathrm{m}^{2}$. Using 5 and $15 \mathrm{mV} / \mathrm{m}$ and 10 and $3 \mathrm{~S}$ for the north and south fields and conductivities we obtain 0.25 and $0.675 \mathrm{~mW} / \mathrm{m}^{2}$. We take $100 \mathrm{~km}$ as the latitudinal regonance width in the ionosphere and $2000 \mathrm{~km}$ as a conseryative

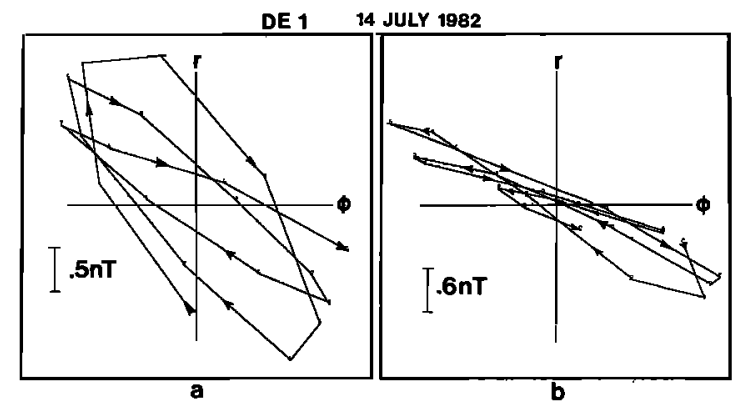

Fig. 13. (a,b)Polarization hodograms of two intervals during the 44-s wave enhancement. Polarization sense is right hand for the first interval and nearly linear for the second. 


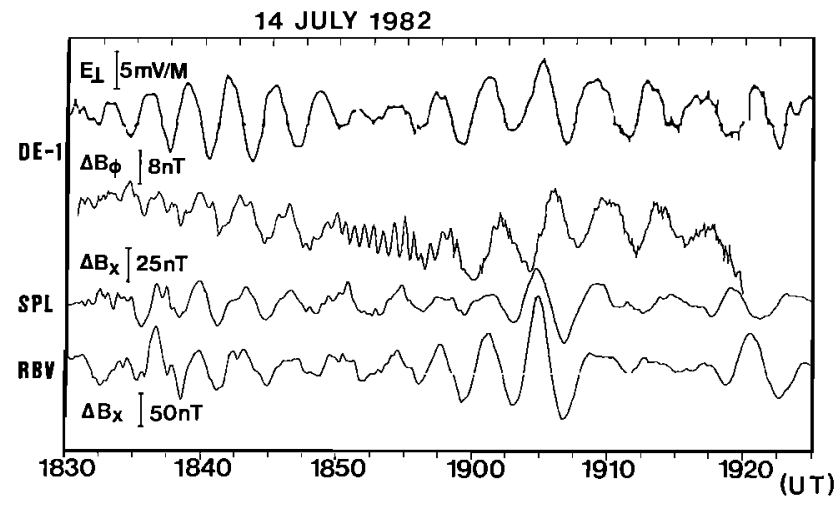

Fig. 14. The electric and magnetic field at DE- 1 and the northward magnetic field at Siple and Roberval from 1830 to 1925 UT. Note that Siple and Roberval $B_{x}$ are in phase from 1835 to 1910 UT.

estimate of the azimuthal extent of the wave (azimuthal separation between $D E-1$ and Siple in the ionosphere is about $1000 \mathrm{~km}$ near $1900 \mathrm{UT}$ ). The power dissipation rate near Roberval and Siple is $50 \mathrm{MW}$ and $135 \mathrm{MW}$.

Unlike the long duration (1-3 hours) toroidal waves seen with the STARE radar by Walker et al. [1979], these waves occur in short bursts like the transient waves (not more than 5 cycles in duration) described by Poulter and Nielsen [1982]. Events 1 and 3 start at the same time at Siple, DE- 1 and Roberval, grow in amplitude, and then decay. The total duration is about $20 \mathrm{~min}$ in each case. Event 3 decays more rapidly at Siple and Roberval than at DE-1. Another 200 s event may start at 1920 UT but only lasts for two cycles. The short duration of these transient eventa implies greater damping than in the long duration events described by Walker et al. [1979].

The fact that $B_{\phi}$ at $D E-1$ and $B_{x}$ at Siple and Roberval remain in phase from 1835 to 1850 UT (Figure 14) implies that there is very little azimuthal variation of the wave phase over $30^{\circ}$ of longitude. The wave has a long azimuthal wavelength. As a limit the $30^{\circ}$ separation corresponds to one quarter azimuthal wavelength or more, equivalent to an azimuthal wave number of 3 or less. The possibility that the zero phase difference is because the separation corresponds to an integral number of much shorter wave lengths seems unlikely, particularly since the separation distance is steadily decreasing.

The brief enhancement in $B_{\phi}$ of the 44-s waves while they are relatively weak in $E_{r}$ is interesting. The 44-s wave may be a fifth harmonic of the 200-s wave. Lin and Cahill [1976] observed the third, fifth, and seventh harmonics of a $500 \mathrm{~s}$ fundamental that started with a compression of the magnetosphere. Harmonics up to the ninth, sometimes without the fundamental apparent, have been observed in ATS 6 records by Takahashi and McPherron [1982]. They do not report observing a single isolated high harmonic however. The fifth harmonic has a node in $B_{\phi}$ at the equator and an antinode several degrees north of the equator. This point would be a node for field displacement, plasma velocity, and $E_{r}$, which could explain the low $E_{r}$ amplitude observed.
The compressional pulsation that starts at 1832 UT is not a pure compressional wave. The phase relation between $B$ and $I$ is not correct. In addition, in Figure 3 a strong $B_{r}$ pulsation close to the equator indicates a transverse component and also suggests it may be a second harmonic as does the 120-s period. In Figure 3 the $\Delta B_{r}$ record or in Figure 2 the $I$ record allow us to follow the field line direction in the meridian plane. At 1834 UT the field direction is pointing more toward the earth than for an undistorted field line ( $I$ greater than for the model). $\Delta B_{r}$ increases until 1835 UT when the field is pointing more away from the earth than at 1834. By 1836 UT it has again returned to a more earthward direction. If the field line motion was a fundamental mode the 1835 direction, for the northern hemisphere, would correspond to a field line displacement toward the earth as can be seen by reference to Figure 8 . In this case let the left side of each sketch in Figure 8 represent the earthward direction. Also let $B_{\phi}$ become $B_{r}$ and $E_{r}$ become $-E_{z}$. If the motion was in a second harmonic mode the 1835 direction would correspond to a northern hemisphere displacement away from the earth. The electric field $E_{z}$ record resolves this ambiguity. Between 1834 and 1835 UT, for the fundamental mode, the field line would be moving inward and $E_{z}$ would be negative or westward. Instead $E_{z}$ has a positive, eastward, peak, and the field line velocity is outward. The first two transverse pulsations appear, therefore, to be a second harmonic field line motion in the magnetic meridian plane. In Figure 3, for the first two pulsations, $\Delta B_{r}$ leads $\Delta B_{\theta}$ in phase by about 90 so the compressional and transverse parts of the initial pulsation are coupled into an elliptical polarization in the magnetic meridian plane. Looking east into that plane the disturbance vector describes a clockwise polarization hodogram. There is a phase shift in the $\Delta B_{T}$ record at 1836 UT and at about 1838 UT a positive $\Delta B_{r}$ pulse indicates the field line is again pointing more away from the earth as at 1835 UT. A positive $E_{z}$ pulsation precedes this so again there was a second harmonic mode oscillation. After 1838 UT the $\Delta B_{r}$ and $\Delta B_{\theta}$ records are too erratic to analyze. One more clear $\Delta B_{r}$ and $\Delta B_{\theta}$ pulsation occurs from 1900 to $1906 \mathrm{UT}$, this time at the 240-s period of the transverse $\Delta B_{\phi}$ oscillation. The $\Delta B_{r}$ pulsation again leads $\Delta B_{\theta}$ by $90^{\circ}$. This time it appears that a westward electric field pulation with field line motion toward the earth, precedes the positive $\Delta B_{r}$ peak at 1901 UT indicating that the oscillation is now a fundamental mode.

The basic time structure of the long-period waves at $\mathrm{DE}-1$, the beginning of the waves, the increase in amplitude and the decay of the waves, is clearly due to time variation of the wave source and not due to the satellite passing radially, azimuthally, or along the field line, into regions with different wave properties. This is because the same structure is seen at the fixed ground locations. The entire oscillating field shell starts and stops oscillating at the times indicated by the $D E-1$ record. Without the ground data one might, for example, conclude the decrease in amplitude at 1852 UT was caused by movement from one resonant shell to another. 
Conclusions

A complex series of toroidal, half-wave fundamental resonant oscillations of magnetospheric field shells near $L=4.5$ have been observed by electric and magnetic instruments on $\mathrm{DE}-1$ and by ground magnetometers at opposite ends of the same magnetic shells. For the first event Siple, DE-1 and Roberval all appear to be on the high L value, high latitude side of the resonant oscillation. This conclusion is based on the statistical evidence about waves believed due to a Kelvin-Helmholtz boundary wave source [Samson et a1., 1971; Fukunishi and Lanzerotti, 1974]. The third event is more complex. Siple and Roberval are on the high-latitude and low-latitude side of a 220-s resonant oscillation, while $\mathrm{DE}-1$ is on the low $L$ value side of a 240-s oscillation.

At least the first event and probably also the third haye a long azimuthal wavelength, greater than 120 in longitude. The third event starts with Siple, DE-1, and Roberval $B_{x}$ in phase suggeating a common source although there are at least two different resonant shells. Amplitudes and phases of the $E$ and $B$ field wave components near the equator and the $B$ components on the ground are in agreement with toroidal model calculations.

The source of the first event must be related to the initial rapidly damped, compressional pulsation. Identification of the transverse part of this initial pulsation as a second harmonic mode requires a source that is not symmetric about the equator. Singer et al. [1982] have invoked a bounce resonant oscillation of particles along the field line to explain lack of symmetry about the equator of similar second harmonic waves. The abrupt initial decrease in field magnitude also suggesta a sudden local increase in ring current particles as seen in events observed earlier [Brown et al., 1966; Barfield et al., 1972]. Ring current particles are symmetric about the equator, however. The third event has no such compressional wave precursor. In this event a compressional pulation develops near the maximum amplitude time of the transverse wave. In each case rapid damping must be caused by the dark, low conductivity, southern ionosphere.

The 44-s waves are more limited in azimuthal extent or have a higher azimuthal wave number. They correspond in period to the 5 th harmonic of the $\sim 200-s$ waves and this suggests that they have the same source as the $\sim$ 200-s waves. Although these pulsations are complex in time structure and in the occurrence of multiple periods such toroidal events are common in the $\mathrm{DE}-1$ data. We have seen waves of comparable or smaller amplitudes with periode from 10 to $300 \mathrm{~s}$ in about half of the equatorial passes studied.

Acknowledgments. This work was made possible through the technical, as well as financial support, of the DE Project Office at Goddard Space Flight Center and the National Science Foundation's Diviaion of Polar Programs. The University of Minnesota was supported in this work by the NASA through contract NAS 5-25389 and by the NSF through grant DPP-8120957. The University of New Hampahire and the Augsburg College contributions were supported also by NSF grant DPP-
8120957. A NASA contract NAS 5-25690 supported the University of Iowa work.

The Editor thanks J. N. Barfield and another referee for their assistance in evaluating this paper.

\section{References}

Allan, W., Phase variation of ULF pulsations along the geomagnetic field line, Planet. Space Sci., $30,339,1982$.

Allan, W., and F. B. Knox, A dipole field model for axisymmetric Alfvén waves with finite ionosphere conductivities, Planet. Space Sci., 27, 79, 1979a.

Allan, W., and F. B. Knox, The effect of finite ionosphere conductivities on axisymetric toroidal Alfvén wave resonances, Planet. Space Sci., 27, 939, 1979b.

Barfield, J. N., R. L. McPherron, P. J. Coleman, and D. J. Southwood, Storm associated Pc 5 micropulsation events observed at synchronous equatorial orbit, J. Geophys. Res., 77, 143, 1972.

Brown, W. L., L. J. Cahill, L. R. Davis, C. E. McIlwain, and C. S. Roberts, Acceleration of trapped particles during a magnetic storm on April 18, 1965, J. Geophys. Res., 71, 153, 1966.

Cauffman, D. P., and N. C. Maynard, A model of the effect of the satellite photosheath on a double floating probe system, J. Geophys. Res., 79, $2427,1974$.

Chen, L., and A. Hasegawa, A theory of long-period magnetic pulsations, 1 , Steady state excitation of field line resonance, J. Geophys. Res., 79, $1024,1974$.

Engebretson, M. J., and L. J. Cahill, Jr., Pc 5 pulsations observed during the June 1972 geomagnetic storm, J. Geophys. Res., 86, 5619, 1981.

Farthing, W. H., M. Sugiura, B. G. Ledley, and L. J. Cahill, Jr., Magnetic field observations on DE-A and -B, Space Sci. Ingtrum., 5, 551, 1981.

Fukunishi, H., and L. J. Lanzerotti, ULF pulsation evidence of the plasmapause, 2, Polarization studies of $\operatorname{PC} 5$ and 4 pulsations near $L=4$ and at a latitude network in the conjugate region, J. Geophys. Res., 79, 4632, 1974.

Greenwald, R. A., and A. D. M. Walker, Energetics of long period resonant hydromagnetic waves, Geophy s. Res. Lett., 7, 745, 1980.

Hasegawa, A., Drift mirror instability in the magnetosphere, Phys. Fluids, 12, 2642, 1969.

Hughes, W. J., and D. J. Southwood, The screening of micropulsation signals by the atmosphere and ionosphere, J. Geophys. Res., 81, 3234, 1976a.

Hughes, W. J., and D. J. Southwood, An illustration of modification of geomagnetic pulsation structure by the ionosphere, J. Geophys. Res., $81,3241,1976 b$.

Hughes, W. J., R. L. McPherron, and J. N. Barfield, Geomagnetic pulsations observed simultaneously on three geostationary satel1ites, J. Geophys.Res., 83, 1109, 1978.

Lange 1, R. A., R. J. Estes, G. D. Mead, G. B. Fabiano, and E. R. Lancaster, Initial geomagnetic field model for MAGSAT vector data, Geophys. Res. Lett., 7, 793, 1980.

Lin, C. C., and L. J. Cahill, Jr., Pc 4 and Pc 5 
pulsations during storm recovery, J. Geophys. Res., 81, 1751; 1976.

Patel, V. L., and L. J. Cahill, Jr., Evidence of hydromagnetic waves in the earth's magnetosphere and of their propagation to the earth's surface, Phys. Rev. Lett., 12, 213, 1964.

Poulter, E. M., and E. Nielsen, The hydromagnetic oscillation of individual shells of the geomagnetic field, J. Geophys. Res., 87, 10432, 1982.

Sapson, J. C., J. A, Jacobs, and G. Rostoker, Latitude dependent characteristics of longperiod geomagnetic micropulsations, J. Geophys. Res., 76, 3675, 1971 .

Shawhan, S. D., D. A. Gurnett, D. I. Odem, R. A. Helliwell, and C. G. Park, The plagma wave and quasi-static electric field instrument (PWI) for Dynamics Explorer-A, Space Sci. Instrum., 5, 535, 1981 .

Singer, H. J., W. J. Hughes, and C. T. Russe11, Standing hydromagnetic wayes observed by ISEE 1 and 2: Radial extent and harmonic, J. Geophys. Res., 87, 3519, 1982.

Southwood; D. J., Some features of field line resonances in the magnetosphere, Planet. Space Sci., 22, 483, 1974 .

Sugiura, $\bar{M}_{*}$, and D. J. Poros, A magnetospheric field model incorporating the 0 GO-3 and 5 magnetic field observations, Planet. Space Sci., 21, 1763, 1973.

Sugiura, $\bar{M}_{i}$, and C. R. Wilson, Oscillation of the geomagnetic field lines and associated magnetic perturbations at conjugate points, J. Geophys. Res., 69, 1211, 1964 .

Takahashi, K., and R. L. McPherron, Harmonic structure of Pc 3-4 pulsations, J. Geophys. Res., 87, 1504, 1982.

Taylor, W. W. L., B. K. Parady, P. B. Lewis, R. L. Afnoldy, and L. J. Cahill, Jr., Initial results from the search coil magnetameter at siple, Anţarçtica, J. Geophy8. Res., 80, 4762, 1975.

Walker, A. 'D. M., R. H. Greenwald, W. F. Stuart, and $C$. H. Green, STARE auroral radar obaervations of Pc 5 geomagnetic pulsations, J. Geophys. Res., 84, 3373, 1979.

R. I. Arnoldy, Department of Physics, Demeritt Hall, Univeraity of New Hampshire, Durham, NH 03824 .

L. J. Cahill and N. G. Lin, School of Physics and Astronomy, University of Minnesota, 100 Union Street S.E., Minneapolis, MN 55455.

M. J. Engebretson, Department of Physics, Augsburg College, Minneapolig, MN 55404.

B. G. Ledley and M. Sugiura, NASA Goddard Space Flight Center, Greenbe1t, MD 20771.

S. D, Shawhan, Department of Physics and Aatronomy, University of Iowa, Iowa City, IA 52242 .

(Received August 25, 1983; revised December 30,1983 ; accepted January 4, 1984.) 